\title{
Mining hub genes correlated with immune infiltrating level across multiple tumors microenvironment
}

\author{
Ning Zhao
}

Wuhan Union Hospital

Liang Wu

Wuhan Union Hospital

\section{Zili Zhou}

Wuhan Union Hospital

Xudan Zhang

Wuhan Union Hospital

Shengbo Han

Wuhan Union Hospital

\section{Haijun Bao}

Wuhan Union Hospital

Xiaogang Shu ( $\nabla$ shuxiaogang_whuh@163.com )

Wuhan Union Hospital https://orcid.org/0000-0003-1336-1772

\section{Research article}

Keywords: Immune infiltrating level, PTPN6, Tumors microenvironment, Multiple cancers

Posted Date: January 10th, 2020

DOl: https://doi.org/10.21203/rs.2.20604/v1

License: @ (i) This work is licensed under a Creative Commons Attribution 4.0 International License. Read Full License 


\section{Abstract}

Background Previous studies revealed that cancer-associated differentially expressed genes (DEGs) in an independent cancer type are rarely related to the tumorigenesis and metastasis, while the common DEGs across multiple types of cancer may be proved as potential oncogenes or tumor suppressors and extend our understanding. Although tumor-infiltrating lymphocytes (TIL) have been reported to be associated with prognosis in multiple types of cancer, the hub genes regulating immune cells function in different cancer types remain unclear.

Methods To screen for the hub genes regulating immune infiltrating level across multiple tumors microenvironment, the raw data containing RNA sequencing and clinical information from TCGA database and immune scores from ESTIMATE website across 25 cancer types were obtained.

Results Based on the immune scores, all cases were categorized into high-score and low-score groups. KaplanMeier survival analysis demonstrated that a strong correlation was found in six cancer types. The functional enrichment analysis of common DEGs revealed that infection and immune response are the most prominent biological characteristics. Subsequently, the twelve common DEGs with prognostic value were identified as candidate hub genes and were adopted to construct the PPI network. Because of highly interconnected with other hub genes, protein tyrosine phosphatase non-receptor type 6 (PTPN6) was selected as the real hub gene across the six immune-specific tumors.

Conclusion Due to the significant correlation between PTPN6 with tumor-infiltrating immune cells in multiple cancers, PTPN6 may well play a vital role in regulating immune response for tumor development.

\section{Background}

A key factor for tumor cells to evade antitumor immune surveillance lies in the complex immunosuppressive microenvironment is formed through downregulation of tumor antigens and production of immunosuppressive mediators[1, 2]. Great progress has been made in modern immunotherapy by recognizing immune checkpoint [3-5] and adopting tumor vaccine[6-8], which suggested that elucidating the antitumor immunerelated mechanisms is crucial to initiate a clinically meaningful antitumor immune response. However, the cancer-associated differentially expressed genes (DEGs) only in an independent cancer type are rarely reported to be related to the initiation and development of cancer[9]. By contrast, tumor samples from different tissues have been reported to share some common features and may act as oncogenes or tumor suppressors across multiple cancer types[10,11]. Therefore, mining hub genes correlated with immune infiltrating level in multiple cancer types may provide novel immune targets for future immunotherapy.

In the tumor microenvironment, immune cells are the principal types of non-tumor components and have been proposed to be significantly correlated with tumor prognosis[12, 13]. ESTIMATE algorithm is based on single sample Gene Set Enrichment Analysis (GSEA) and generates immune scores via gene expression data from The Cancer Genome Atlas (TCGA) database[14]. To date, immune scores based on ESTIMATE algorithm have been widely adopted to quantify the immune components in multiple types of tumor tissues, including colon cancer[15], breast cancer[16], prostate cancer[17] and glioblastoma multiforme[18]. However, the immune-related 
genes in previous studies are various and rarely have been confirmed to be associated with immune infiltrating level in tumor microenvironment.

In this study, we systematically analyzed the correlation between immune scores and survival prognosis across 25 types of cancer and six cancer types were selected as immune-specific cancers. Subsequently, the common DEGs with prognostic value were identified as candidate hub genes. Protein-protein interaction (PPI) network were then used to screen the real hub gene. Finally, we investigated the relationship between the real hub gene protein tyrosine phosphatase non-receptor type 6 (PTPN6) and immune infiltrating level in tumor microenvironment.

\section{Results}

\section{Identification of immune-specific tumors}

To single out the immune-associated tumors, immune scores across 25 types of cancer and their clinical information from TCGA were obtained. All cases were divided into high and low-score groups based on immune scores. Kaplan-Meier survival analysis in an independent cancer type demonstrated that a strong correlation was found in six cancer types (including lung adenocarcinoma (LUAD), cervical squamous cell carcinoma and endocervical adenocarcinoma (CESC), skin cutaneous melanoma (SKCM), breast invasive carcinoma (BRCA), brain lower grade glioma (LGG) and kidney renal clear cell carcinoma (KIRC)), which were considered as immune-specific tumors. Further analysis demonstrated that four types of cancer had a favorable overall survival in high-score group (LUAD:1499d vs. 1194d, $P=0.0139$ (Fig. 2A); CESC: $4086 d$ vs. 3046d, $P=0.0493$ (Fig. 2B); SKCM: 3564d vs. 1910d, P < 0.0001 (Fig. 2C); BRCA: 6593d vs. 3873d, $P=0.0255$ (Fig. 2D)), while the remaining two tumors show a poorer prognosis in high-score group (LGG: 2000d vs. 2907d, $P=0.0151$ (Fig. 2E); KIRC: 1980 d vs. 2651 d, $P=0.0130$ (Fig. 2F)). For the convenience of subsequent description, we name these two groups positive and negative subgroups, respectively.

\section{Identification of the common DEGs in immune-specific tumors}

Based on the criterion of $|\log 2(\mathrm{FC})|>2$ and adj. $\mathrm{P}$ value $<0.05$, we compared gene expression profiles between cancer and normal tissue samples in positive and negative subgroups, respectively. In the positive subgroup, we found 2877 DEGs in LUAD, 2071 in CESC, 2467 in SKCM, 2846 in BRCA, respectively. In the negative subgroup, we found 3351 DEGs in LGG and 1947 in KIRC, respectively. The total number of common DEGs is 602 (455 upregulated (Fig. 3A) and 147 down-regulated (Fig. 3B)) and 713 (450 up-regulated (Fig. 3C) and 263 downregulated (Fig. 3D)) in positive and negative subgroup, respectively.

To gain insight into the biological importance of common DEGs, we performed Gene Ontology (GO) analysis and Kyoto Encyclopedia of Genes and Genomes (KEGG) pathways analyses in both subgroups. The top five most significant $G O$ terms $(P<0.05)$ and the top ten most significant KEGG pathways $(P<0.05$ and $>20$ enriched genes) are presented. The GO of the common DEGs in both subgroups were primarily enriched in the activation and regulation of immune response. However, the major mechanism involved in regulating immune response is cell surface receptor signaling and lymphocyte mediated immunity in positive subgroup (Fig. 3E), but it is neutrophil mediated immunity in negative subgroup (Fig. 3F). In addition, the majority of the top ten KEGG 
pathways are shared in both subgroups and enriched in infection and immune response, including Epstein-Barr virus infection, phagosome, antigen processing and presentation, staphylococcus aureus infection, viral myocarditis, allograft rejection and graft-versus-host disease (Fig. 3H-G).

\section{Identification of hub genes}

All DEGs in six cancer types were subjected to univariate Cox proportional hazards regression analysis and a total of $131,214,445,238,454$ and 295 DEGs in LUAD, CESC, SKCM, BRCA, LGG and KIRC were recognized to be associated with their survival prognosis. In the positive and negative subgroups, we found 30 and 213 common DEGs with prognostic value, respectively (Fig. 4A-B). The 12 common DEGs with prognostic value in both subgroups were subsequently extracted and were selected as candidate hub genes, including PTPN6, GRB2 related adaptor protein 2 (GRAP2), coronin 1A (CORO1A), killer cell lectin like receptor C1 (KLRC1), Janus kinase 2 (JAK2), RAS protein activator like 3 (RASAL3), drebrin like (DBNL), mitogen-activated protein kinase 1 (MAP4K1), TBC1 domain family member 10C (TBC1D10C), transcription elongation factor B (TCEB1), suppressor of cytokine signaling 1 (SOCS1) and Rho GTPase activating protein 9 (ARHGAP9) (Fig. 4C). To identify the real hub gene, the candidate hub genes proceed to construct the PPI network. Because of highly interconnected with other nodes, PTPN6 was selected as the real hub gene, which has a significant correlation with the KLRC1, JAK2, RASAL3, DBNL, GRAP2 and CORO1A (Fig. 4D).

\section{Validation of the real hub gene}

To validate the differential expression of the real hub genes on translational level in immune-specific tumors, the immunohistochemistry staining was obtained from HPA database. The results demonstrated that the expression level of PTPN6 was significantly upregulated in tumors compared to the normal samples (Fig. 5A-F). In addition, the prognostic role of PTPN6 was further confirmed using TCGA data. Kaplan-Meier survival analysis showed that high expression level of PTPN6 was significantly correlated with better overall survival in LUAD ( $=$ $0.0303), \operatorname{CESC}(P=0.0386)$, SKCM $(P \otimes 0.0001)$ and BRCA $(P=0.0164)$, but with poorer prognosis in LGG $(P=0.0060)$ and KIRC $(P=0.0116)(F i g .6 A-F)$. These findings were consistent with the correlation between immune scores and survival prognosis.

\section{Correlation analysis between PTPN6 and immune infiltrating level in tumor microenvironment}

To quantify the interactive relationships between PTPN6 and immune infiltrating level in tumor microenvironment, the correlation based on the coefficient of correlation $\mathrm{R}$ was divided into three types as follows: (1) $0 \leq r<0.3$ (1 points); (2) $0.3 \leq r \leq 0.5$ (2 points); (3) $r>0.5$ (3 points). First of all, a significantly negative association between PTPN6 and tumor purity was found in all six cancer types (LUAD: $r=-0.335, P=$ 1.96e-14; CESC: $r=-0.168, P=5.08 \mathrm{e}-03$; SKCM: $r=-0.711, P=1.33 e-71 ;$ BRCA: $r=-0.154, P=1.03 e-06 ;$ LGG: $r=$ $-0.366, P=1.23 e-16 ;$ KIRC: $r=-0.211, P=4.73 e-06)$. Secondly, the expression level of PTPN6 and tumor infiltrating immune cells were highly correlated in SKCM (13 scores) (Fig. 7C) and LGG (14 scores) (Fig. 7E), which CD8+ T cells was independent of PTPN6 in LGG $(r=0.032, P=0.484)$. In addition, PTPN6 has a moderate 
correlation with immune infiltrating level in LUAD (9 scores) (Fig. 7A) and CESC (11 scores) (Fig. 7B), but a weak association was found in BRCA (4 scores) (Fig. 7D) and KIRC (7 scores) (Fig. 7F). It is remarkable that CD8+ T cells $(r=-0.077, P=0.0167)$ and macrophage $(r=-0.129, P=5.13 e-05)$ were negative correlated with PTPN6 in BRCA.

To investigate the interplay between PTPN6 and tumor infiltrating immune cells, the co-expression between PTPN6 and immune marker sets of diverse immune cells was further analyzed after the correlation adjustment by purity. As is shown in table 1, PTPN6 expression was significantly correlated with most immune marker sets of diverse immune cells. the immune marker highly correlated with PTPN6 in various specific immune cells was further explored. The results show that PTPN6 was significantly correlated with CD19 and CD79A in B cell, CD8A and CD8B in CD8+ T cell, TBX21, STAT6 and IFNG in CD4+ T cell, CD68 and IL-10 in tumor-associated macrophage, ITGAM and CCR7 in neutrophils, ITGAM and HLA-DPB1 in dendritic cell (Fig.8A-F). Interestingly, PTPN6 was found to be highly interplayed with PD-1 in T cell exhaustion across six cancer types, which suggests that PTPN6 may play a vital role in regulating immune checkpoint.

\section{Discussion}

Great success has been made in applying immunotherapy to hematologic malignancies[19], while unique challenges are faced in solid tumors because of the immunosuppressive tumor microenvironment[20]. The tumor microenvironment contains multiple components (such as suppressive cells, inhibitory ligands and soluble factors) that facilitate evasion of antitumor immune responses in solid tumors. Previous reports have provided elegant analysis on how the activation of tumor-intrinsic genes shapes tumor microenvironment[21, 22]. With the rapid development of third-generation sequencing technology, an increasing number of available databases have been used to mine candidate genes regulating immune infiltration in an independent cancer type, while immune-associated genes in multiple cancer types have not been reported[10, 11]. In this study, we focused on genes characteristic of tumor microenvironment, which in turn affect the development of cancer and hence contribute to patients' overall survival.

Six selected cancer types (including LUAD, CESC, SKCM, BRCA, LGG and KIRC) were found to be significantly correlated with immune infiltrating level in tumor microenvironment. Twelve common DEGs with prognostic value were adopted to construct PPI network and identify potential hub genes that are associated with tumor infiltrating immune cells across multiple cancer types. The results show that most of them are crucial to immune cell signaling and immune responses. For example, GRAP2[23], DBNL[24], TBC1D10C[25], PTPN6[26, 27] and SOCS1[28] has been reported to be involved in the process of proliferation and activation, cytokine secretion and cell cycle regulation in T cells. In addition, data from experimental mice and clinical observations have unraveled that KLRC1[29], RASAL3[30] and MAP4K1[31] may play a vital role in controlling the magnitude of inflammatory responses through multiple signaling events in autoimmune diseases or tumorigenesis, and JAK2[32, 33] and Coro1 $\mathrm{A}$ [34] represent important novel players with key functions in polymorphonuclear neutrophils trafficking and PD-1 ligand transcription in innate and adaptive immunity. Although there have been no studies to provide evidence for TCEB1 and ARHGAP9 with immune-infiltrating level in tumor microenvironment, they exhibit an accordant up-expression pattern in the cancer tissues in our study.

Because of highly interconnected with other nodes in the PPI network, PTPN6 was considered to be the most valuable one related to immune response. A systematic correlation analysis uncovered that the expression level 
of PTPN6 was significantly associated with immune infiltrating immune cells and marker sets of diverse immune cells, among which CD19 and CD79A in B cell, CD8A and CD8B in CD8 + T cell, TBX21, STAT6 and IFNG in CD4 + T cell, CD68 and IL-10 in tumor-associated macrophage, ITGAM and CCR7 in neutrophils, ITGAM and HLA-DPB1 in dendritic cell, PD-1 in T cell exhaustion were found to be significantly co-expressed with PTPN6. Previous reports have disclosed the role of PTPN6 in regulating CD8 + T cell activation and responsiveness by inhibiting immune checkpoint and increasing $T$ cell receptor affinity to enhance $T$ cell-mediated immunity[26, 35]. Moreover, Zawada et al. performed a GO enrichment analysis of monocyte heterogeneity, which suggests that PTPN6 was significantly associated with monocyte activation[27]. However, the relationship between PTPN6 and other marker sets of diverse immune cells remains unclear. Therefore, our results may provide additional data in decoding the complex interaction of tumor and tumor microenvironment.

In conclusion, our results demonstrated that immune scores are significantly correlated with overall survival in six cancer types, including LUAD, CESC, SKCM, BRCA, LGG and KIRC. No significant differences between positive and negative subgroups were observed in the functional enrichment analysis of common DEGs. Subsequently, the twelve common DEGs with prognostic value were found to be significantly correlated with immune infiltrating level in tumor microenvironment. Finally, PTPN6 was selected as the hub gene regulating tumor infiltrating immune cells and marker sets of diverse immune cells by highly co-expressing with KLRC1, JAK2, RASAL3, DBNL, GRAP2 and COR01A. In summary, the further investigation of these genes may lead to novel insights into the potential association of tumor microenvironment with tumors and provide novel targets for tumor immunotherapy.

\section{Materials And Methods}

\section{Dataset}

A workflow of our study is presented in Figure 1. Immune scores based on ESTIMATE algorithm across 25 types of cancer were downloaded from ESTIMATE website (https://bioinformatics.mdanderson.org/publicsoftware/estimate/). The raw data containing RNA sequencing and clinical information were obtained from The Cancer Genome Atlas (TCGA) repository website (https://portal.gdc.cancer.gov/). This study was approved by the ethics committee of Wuhan union hospital.

\section{Screening for immune-specific cancers}

All patients in an independent cancer type were divided into high and low-score groups according to immune scores. Kaplan-Meier survival analysis was done to screen for tumors that are significantly correlated with survival prognosis.

\section{Identification of DEGs in immune-specific cancers}

The R 'limma' Bioconductor package was used to screen the DEGs between tumor and normal samples under the threshold of $|\log 2(\mathrm{FC})|>2$ and adj. $\mathrm{P}$ value $<0.05$. 


\section{Functional enrichment analysis of DEGs}

DEGs were uploaded to the Database for Annotation, Visualization and Integrated Discovery (DAVID) (https://david.ncifcrf.gov/) to gain insight into the biological themes, particularly GO categories and KEGG pathways. Adj. $\mathrm{P}<0.01$ was set as the cut-off criterion.

\section{The identification and validation of hub gene}

Venn diagram of DEGs across six types of cancer was performed to screen for the common DEGs.

Subsequently, the R 'survival' package was adopted to perform Kaplan-Meier survival analysis according to the expression levels of DEGs. The common DEGs with prognostic value were selected as candidate hub genes. The PPI network of common DEGs was then retrieved from STRING database and reconstructed using Cytoscape software. The node that is highly interconnected with other candidate hub genes were considered to be the real hub gene. The Human Protein Atlas (HPA) (https://www.proteinatlas.org/) were used to validate the differential expression of hub gene between tumor and normal samples on translational level.

\section{Correlation analysis between hub genes and immune infiltrating level in tumor microenvironment}

The correlation between hub genes and immune infiltrating level in tumor microenvironment was analyzed in TIMER website (https://cistrome.shinyapps.io/timer/). In addition, we further assessed the correlation between hub gene and immune marker sets of various immune cells.

\section{Statistical Analysis}

Statistical analysis and visualization. Statistical analysis was performed using R-3.6.1. Most of the visualizations were also presented by $\mathrm{R}$, except for the survival analysis and the network visualization, where the Kaplan-Meier Plotter and Cytoscape v3.3.0 tools were used. For the survival analysis, we chose recommended parameters from the web server to analyze the association between a queried gene and the survival time.

\section{Declarations}

\section{Acknowledgement:}

The authors gratefully acknowledge contributions from the CGGA network and the TCGA Network.

\section{Authors' contributions}


Ning Zhao, Liang Wu, and Xiaogang Shu conceived and designed the study. Ning Zhao, Zili Zhou, Xudan Zhang, Shengbo Han, and Haijun Bao collected and analyzed data. Ning Zhao wrote the paper. Zili Zhou and Xiaogang Shu reviewed and edited the manuscript. All authors read and approved the manuscript.

\section{Funding:}

This work was supported by a grant from National Natural Science Foundation of China $(81772581,81271199$ and 81470789)

\section{Availability of data and materials}

The raw data on patients with HCC containing RNA-seq transcriptome data and clinical information were obtained from The Cancer Genome Atlas (TCGA) repository website.

\section{Ethics approval and consent to participate}

Not applicable.

\section{Consent for publication}

Not applicable

\section{Conflict of interest:}

The authors declared that they have no competing interests.

\section{References}

[1] Ma Y, Pitt JM, Li Q, Yang H. The renaissance of anti-neoplastic immunity from tumor cell demise. Immunological reviews. 2017;280(1):194-206.

[2] Huber V, Camisaschi C, Berzi A, Ferro S, Lugini L, Triulzi T, et al. Cancer acidity: An ultimate frontier of tumor immune escape and a novel target of immunomodulation. Seminars in cancer biology. 2017;43:74-89.

[3] Pardoll DM. The blockade of immune checkpoints in cancer immunotherapy. Nature reviews Cancer. 2012;12(4):252-264.

[4] Nishino M, Ramaiya NH, Hatabu H, Hodi FS. Monitoring immune-checkpoint blockade: response evaluation and biomarker development. Nature reviews Clinical oncology. 2017;14(11):655-668. 
[5] Xu-Monette ZY, Zhou J, Young KH. PD-1 expression and clinical PD-1 blockade in B-cell lymphomas. Blood. 2018;131(1):68-83.

[6] Li L, Goedegebuure SP, Gillanders WE. Preclinical and clinical development of neoantigen vaccines. Annals of oncology : official journal of the European Society for Medical Oncology. 2017;28(suppl_12):xii11-xii17.

[7] Reardon DA, Mitchell DA. The development of dendritic cell vaccine-based immunotherapies for glioblastoma. Seminars in immunopathology. 2017;39(2):225-239.

[8] Loffler MW, Chandran PA, Laske K, Schroeder C, Bonzheim I, Walzer M, et al. Personalized peptide vaccineinduced immune response associated with long-term survival of a metastatic cholangiocarcinoma patient. Journal of hepatology. 2016;65(4):849-855.

[9] lyer MK, Niknafs YS, Malik R, Singhal U, Sahu A, Hosono Y, et al. The landscape of long noncoding RNAs in the human transcriptome. Nature genetics. 2015;47(3):199-208.

[10] Cabanski CR, White NM, Dang HX, Silva-Fisher JM, Rauck CE, Cicka D, et al. Pan-cancer transcriptome analysis reveals long noncoding RNAs with conserved function. RNA biology. 2015;12(6):628-642.

[11] Li S, Li B, Zheng Y, Li M, Shi L, Pu X. Exploring functions of long noncoding RNAs across multiple cancers through co-expression network. Scientific reports. 2017;7(1):754.

[12] Ngambenjawong C, Gustafson HH, Pun SH. Progress in tumor-associated macrophage (TAM)-targeted therapeutics. Advanced drug delivery reviews. 2017;114:206-221.

[13] Bremnes RM, Busund LT, Kilvaer TL, Andersen S, Richardsen E, Paulsen EE, et al. The Role of TumorInfiltrating Lymphocytes in Development, Progression, and Prognosis of Non-Small Cell Lung Cancer. Journal of thoracic oncology : official publication of the International Association for the Study of Lung Cancer. 2016;11(6):789-800.

[14] Yoshihara K, Shahmoradgoli M, Martinez E, Vegesna R, Kim H, Torres-Garcia W, et al. Inferring tumour purity and stromal and immune cell admixture from expression data. Nature communications. 2013;4:2612.

[15] Alonso MH, Ausso S, Lopez-Doriga A, Cordero D, Guino E, Sole X, et al. Comprehensive analysis of copy number aberrations in microsatellite stable colon cancer in view of stromal component. British journal of cancer. 2017;117(3):421-431.

[16] Priedigkeit N, Watters RJ, Lucas PC, Basudan A, Bhargava R, Horne W, et al. Exome-capture RNA sequencing of decade-old breast cancers and matched decalcified bone metastases. JCI insight. 2017;2(17).

[17] Shah N, Wang P, Wongvipat J, Karthaus WR, Abida W, Armenia J, et al. Regulation of the glucocorticoid receptor via a BET-dependent enhancer drives antiandrogen resistance in prostate cancer. 2017;6.

[18] Jia D, Li S, Li D, Xue H, Yang D, Liu Y. Mining TCGA database for genes of prognostic value in glioblastoma microenvironment. Aging. 2018;10(4):592-605.

[19] Armand P. Immune checkpoint blockade in hematologic malignancies. Blood. 2015;125(22):3393-3400. 
[20] Lanitis E, Dangaj D, Irving M, Coukos G. Mechanisms regulating T-cell infiltration and activity in solid tumors. Annals of oncology : official journal of the European Society for Medical Oncology. 2017;28(suppl_12):xii18xii32.

[21] Clark CA, Gupta HB, Sareddy G, Pandeswara S, Lao S, Yuan B, et al. Tumor-Intrinsic PD-L1 Signals Regulate Cell Growth, Pathogenesis, and Autophagy in Ovarian Cancer and Melanoma. Cancer research. 2016;76(23):6964-6974.

[22] Codina A, Renauer PA, Wang G, Chow RD, Park JJ, Ye H, et al. Convergent Identification and Interrogation of Tumor-Intrinsic Factors that Modulate Cancer Immunity In Vivo. Cell systems. 2019;8(2):136-151.e137.

[23] Ma W, Xia C, Ling P, Qiu M, Luo Y, Tan TH, et al. Leukocyte-specific adaptor protein Grap2 interacts with hematopoietic progenitor kinase 1 (HPK1) to activate JNK signaling pathway in T lymphocytes. Oncogene. 2001;20(14):1703-1714.

[24] Han J, Shui JW, Zhang X, Zheng B, Han S, Tan TH. HIP-55 is important for T-cell proliferation, cytokine production, and immune responses. Molecular and cellular biology. 2005;25(16):6869-6878.

[25] Patino-Lopez G, Dong X, Ben-Aissa K, Bernot KM, Itoh T, Fukuda M, et al. Rab35 and its GAP EPI64C in T cells regulate receptor recycling and immunological synapse formation. The Journal of biological chemistry. 2008;283(26):18323-18330.

[26] Hebeisen M, Baitsch L, Presotto D, Baumgaertner P, Romero P, Michielin O, et al. SHP-1 phosphatase activity counteracts increased T cell receptor affinity. The Journal of clinical investigation. 2013;123(3):1044-1056.

[27] Zawada AM, Rogacev KS, Rotter B, Winter P, Marell RR, Fliser D, et al. SuperSAGE evidence for CD14++CD16+ monocytes as a third monocyte subset. Blood. 2011;118(12):e50-61.

[28] Yoshimura A, Ito M, Chikuma S, Akanuma T, Nakatsukasa H. Negative Regulation of Cytokine Signaling in Immunity. Cold Spring Harbor perspectives in biology. 2018;10(7).

[29] Rapaport AS, Schriewer J, Gilfillan S, Hembrador E, Crump R, Plougastel BF, et al. The Inhibitory Receptor NKG2A Sustains Virus-Specific CD8(+) T Cells in Response to a Lethal Poxvirus Infection. Immunity. 2015;43(6):1112-1124.

[30] Muro R, Nitta T, Kitajima M, Okada T, Suzuki H. Rasal3-mediated T cell survival is essential for inflammatory responses. Biochemical and biophysical research communications. 2018;496(1):25-30.

[31] Chuang HC, Wang X, Tan TH. MAP4K Family Kinases in Immunity and Inflammation. Advances in immunology. 2016;129:277-314.

[32] Green MR, Monti S, Rodig SJ, Juszczynski P, Currie T, O'Donnell E, et al. Integrative analysis reveals selective 9p24.1 amplification, increased PD-1 ligand expression, and further induction via JAK2 in nodular sclerosing Hodgkin lymphoma and primary mediastinal large B-cell lymphoma. Blood. 2010;116(17):3268-3277.

[33] Ghoreschi K, Laurence A, O'Shea JJ. Janus kinases in immune cell signaling. Immunological reviews. 2009;228(1):273-287. 
[34] Pick R, Begandt D, Stocker TJ, Salvermoser M, Thome S, Bottcher RT, et al. Coronin 1A, a novel player in integrin biology, controls neutrophil trafficking in innate immunity. Blood. 2017;130(7):847-858.

[35] Pao LI, Lam KP, Henderson JM, Kutok JL, Alimzhanov M, Nitschke L, et al. B cell-specific deletion of proteintyrosine phosphatase Shp1 promotes B-1a cell development and causes systemic autoimmunity. Immunity. 2007;27(1):35-48.

\section{Table}

Table 1. Correlation analysis between PTPN6 and markers of immune infiltrating cells in the tumor microenvironment.

\begin{tabular}{|c|c|c|c|c|c|c|c|c|c|c|c|c|c|}
\hline \multirow[t]{2}{*}{ Type } & \multirow{2}{*}{$\begin{array}{l}\text { Gene } \\
\text { Markers }\end{array}$} & \multicolumn{2}{|c|}{ LUAD } & \multicolumn{2}{|c|}{ CESC } & \multicolumn{2}{|c|}{ SKCM } & \multicolumn{2}{|c|}{ BRCA } & \multicolumn{2}{|c|}{ LGG } & \multicolumn{2}{|c|}{ KIRC } \\
\hline & & Cor & $\mathrm{P}$ & Cor & $\mathrm{P}$ & Cor & $\mathrm{P}$ & Cor & $\mathrm{P}$ & Cor & $\mathrm{P}$ & Cor & $\mathrm{P}$ \\
\hline \multirow[t]{2}{*}{ B cell } & CD19 & 0.326 & $<0.001$ & 0.416 & $<0.001$ & 0.392 & $<0.001$ & 0.381 & $<0.001$ & 0.375 & $<0.001$ & 0.463 & $<0.001$ \\
\hline & CD79A & 0.224 & $<0.001$ & 0.4 & $<0.001$ & 0.499 & $<0.001$ & 0.332 & $<0.001$ & 0.478 & $<0.001$ & 0.505 & $<0.001$ \\
\hline \multirow{2}{*}{$\begin{array}{l}\text { CD8+ T } \\
\text { cell } \\
\end{array}$} & CD8A & 0.285 & $<0.001$ & 0.542 & $<0.001$ & 0.732 & $<0.001$ & 0.358 & $<0.001$ & 0.043 & 0.349 & 0.593 & $<0.001$ \\
\hline & CD8B & 0.281 & $<0.001$ & 0.479 & $<0.001$ & 0.718 & $<0.001$ & 0.327 & $<0.001$ & 0.15 & 0.001 & 0.613 & $<0.001$ \\
\hline \multirow{7}{*}{$\begin{array}{l}\text { CD4+ T } \\
\text { cell }\end{array}$} & TBX21 & 0.385 & $<0.001$ & 0.553 & $<0.001$ & 0.739 & $<0.001$ & 0.436 & $<0.001$ & 0.298 & $<0.001$ & 0.437 & $<0.001$ \\
\hline & STAT4 & 0.338 & $<0.001$ & 0.435 & $<0.001$ & 0.518 & $<0.001$ & 0.298 & $<0.001$ & -0.29 & $<0.001$ & 0.485 & $<0.001$ \\
\hline & STAT1 & 0.144 & 0.002 & 0.377 & $<0.001$ & 0.289 & 0.003 & 0.142 & $<0.001$ & 0.258 & $<0.001$ & 0.375 & $<0.001$ \\
\hline & IFNG & 0.308 & $<0.001$ & 0.461 & $<0.001$ & 0.537 & $<0.001$ & 0.319 & $<0.001$ & 0.222 & $<0.001$ & 0.609 & $<0.001$ \\
\hline & TNF & 0.32 & $<0.001$ & 0.033 & 0.58 & 0.561 & $<0.001$ & 0.217 & $<0.001$ & 0.379 & $<0.001$ & 0.441 & $<0.001$ \\
\hline & GATA3 & 0.339 & $<0.001$ & 0.15 & 0.013 & 0.335 & $<0.001$ & 0.081 & 0.01 & 0.393 & $<0.001$ & 0.303 & $<0.001$ \\
\hline & STAT6 & 0.318 & $<0.001$ & 0.238 & $<0.001$ & 0.188 & 0.059 & 0.265 & $<0.001$ & 0.471 & $<0.001$ & 0.31 & $<0.001$ \\
\hline \multirow[t]{3}{*}{ TAM } & CCL2 & 0.159 & $<0.001$ & 0.059 & 0.327 & 0.335 & $<0.001$ & 0.215 & $<0.001$ & 0.623 & $<0.001$ & 0.073 & 0.116 \\
\hline & CD68 & 0.461 & $<0.001$ & 0.37 & $<0.001$ & 0.191 & 0.055 & 0.317 & $<0.001$ & 0.884 & $<0.001$ & 0.548 & $<0.001$ \\
\hline & IL10 & 0.228 & $<0.001$ & 0.204 & $<0.001$ & 0.415 & $<0.001$ & 0.243 & $<0.001$ & 0.634 & $<0.001$ & 0.349 & $<0.001$ \\
\hline \multirow[t]{3}{*}{ Neutrophil } & CEACAM8 & 0.17 & $<0.001$ & 0.145 & 0.016 & -0.103 & 0.302 & 0.021 & 0.513 & 0.046 & 0.312 & -0.002 & 0.963 \\
\hline & ITGAM & 0.436 & $<0.001$ & 0.424 & $<0.001$ & 0.656 & $<0.001$ & 0.282 & $<0.001$ & 0.847 & $<0.001$ & 0.522 & $<0.001$ \\
\hline & CCR7 & 0.397 & $<0.001$ & 0.427 & $<0.001$ & 0.528 & $<0.001$ & 0.411 & $<0.001$ & 0.288 & $<0.001$ & 0.45 & $<0.001$ \\
\hline \multirow[t]{7}{*}{$\begin{array}{l}\text { Dendritic } \\
\text { cell }\end{array}$} & $\begin{array}{l}\text { HLA- } \\
\text { DPB1 }\end{array}$ & 0.49 & $<0.001$ & 0.575 & $<0.001$ & 0.712 & $<0.001$ & 0.502 & $<0.001$ & 0.688 & $<0.001$ & 0.672 & $<0.001$ \\
\hline & $\begin{array}{l}\text { HLA- } \\
\text { DQB1 } \\
\end{array}$ & 0.365 & $<0.001$ & 0.469 & $<0.001$ & 0.621 & $<0.001$ & 0.394 & $<0.001$ & 0.536 & $<0.001$ & 0.421 & $<0.001$ \\
\hline & HLA-DRA & 0.437 & $<0.001$ & 0.498 & $<0.001$ & 0.673 & $<0.001$ & 0.388 & $<0.001$ & 0.727 & $<0.001$ & 0.585 & $<0.001$ \\
\hline & $\begin{array}{l}\text { HLA- } \\
\text { DPA1 }\end{array}$ & 0.426 & $<0.001$ & 0.52 & $<0.001$ & 0.686 & $<0.001$ & 0.385 & $<0.001$ & 0.672 & $<0.001$ & 0.566 & $<0.001$ \\
\hline & CD1C & 0.305 & $<0.001$ & 0.308 & $<0.001$ & 0.423 & $<0.001$ & 0.273 & $<0.001$ & 0.428 & $<0.001$ & 0.194 & $<0.001$ \\
\hline & NRP1 & -0.086 & 0.056 & -0.145 & 0.016 & 0.084 & 0.402 & -0.273 & $<0.001$ & 0.153 & $<0.001$ & -0.403 & $<0.001$ \\
\hline & ITGAX & 0.566 & $<0.001$ & 0.417 & $<0.001$ & 0.484 & $<0.001$ & 0.429 & $<0.001$ & 0.755 & $<0.001$ & 0.622 & $<0.001$ \\
\hline \multirow{2}{*}{$\begin{array}{l}\text { T cell } \\
\text { exhaustion }\end{array}$} & PDCD1 & 0.452 & $<0.001$ & 0.599 & $<0.001$ & 0.76 & $<0.001$ & 0.477 & $<0.001$ & 0.588 & $<0.001$ & 0.702 & $<0.001$ \\
\hline & CTLA4 & 0.379 & $<0.001$ & 0.548 & $<0.001$ & 0.196 & 0.048 & 0.371 & $<0.001$ & 0.363 & $<0.001$ & 0.581 & $<0.001$ \\
\hline
\end{tabular}

\section{Figures}


Downloading the immune scores, mRNA expression data and clinical information across 25 types of cancer

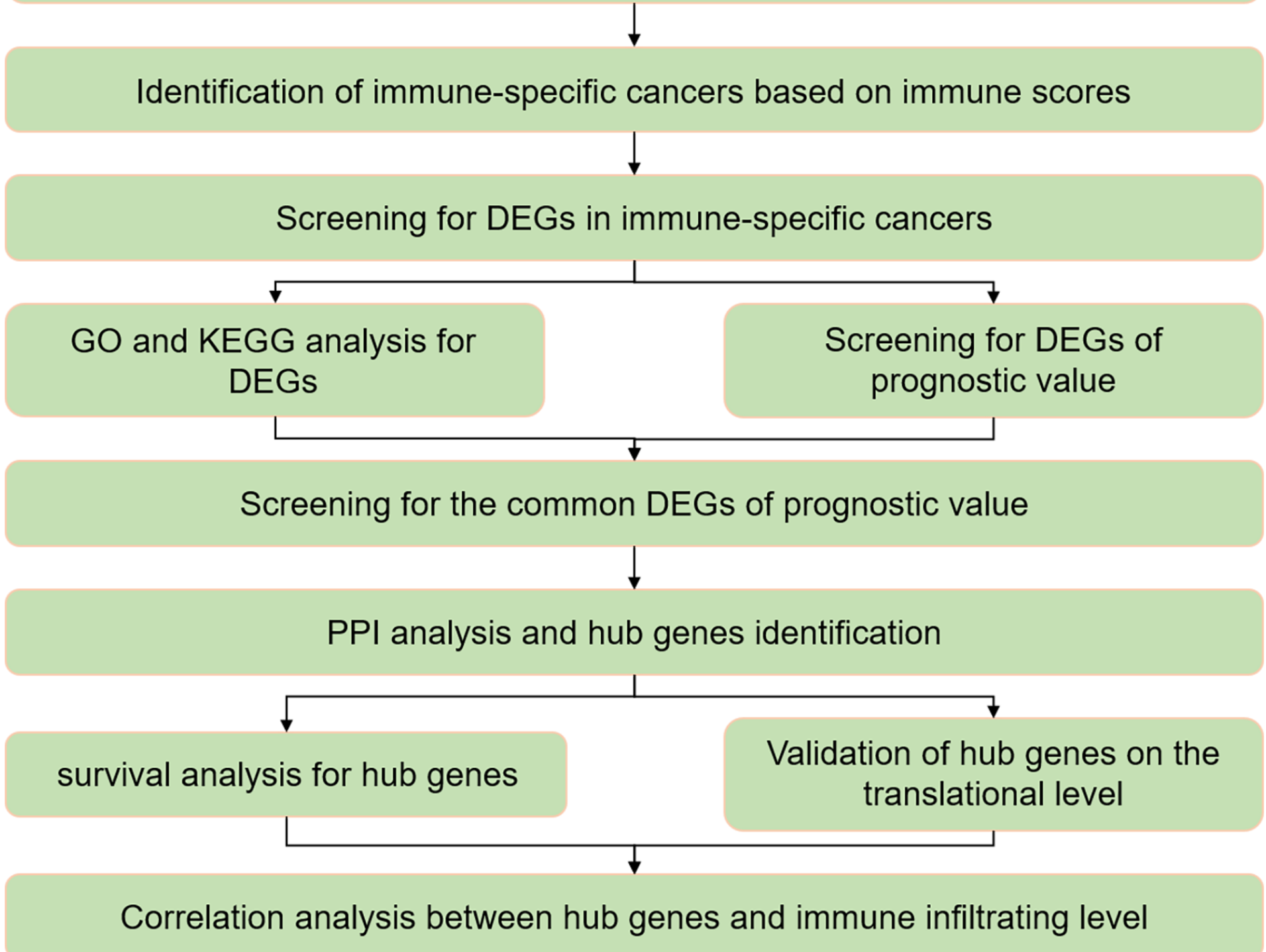

Figure 1

Work flow of the present study. DEG, differentially expressed gene; GO, gene ontology; KEGG, Kyoto Encyclopaedia of Genes and Genomes; PPI, protein-protein interaction; 
A

LUAD

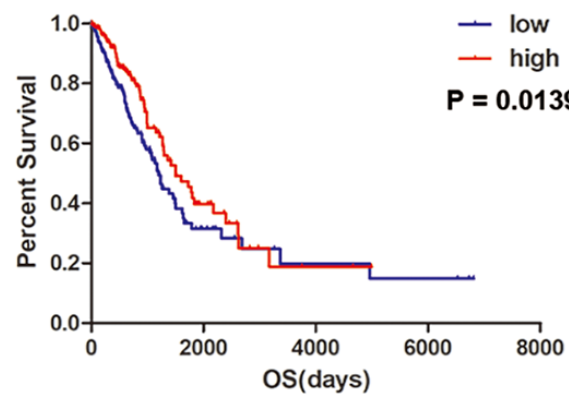

D

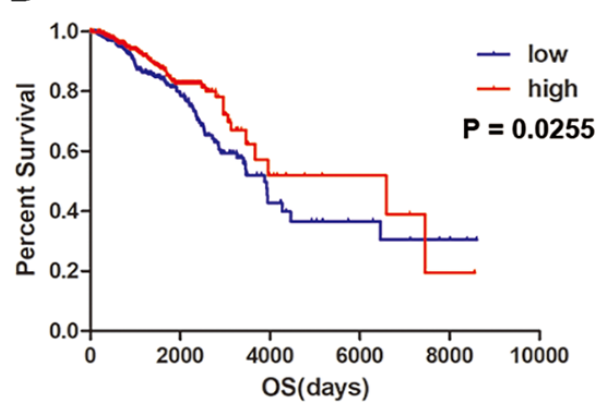

B

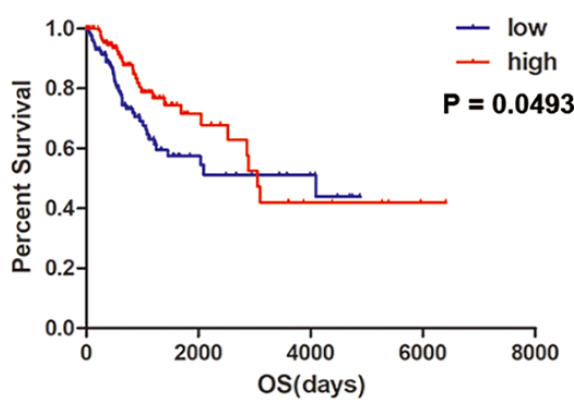

E

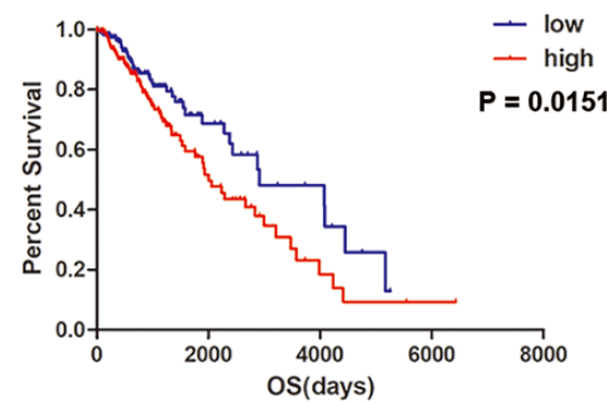

C

SKCM

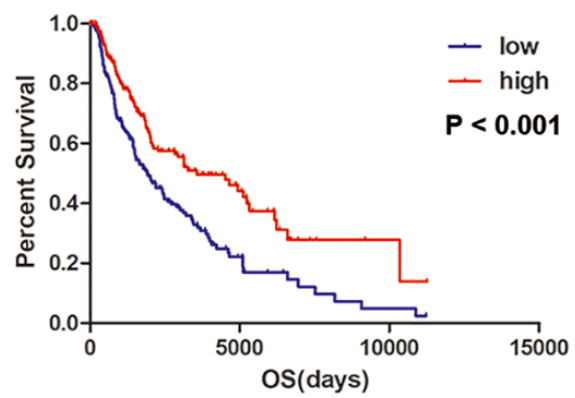

F KIRC

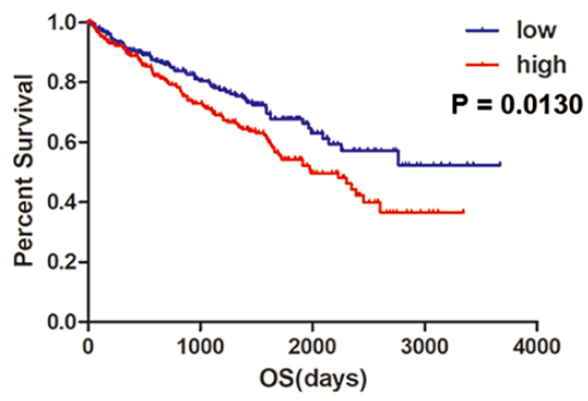

\section{Figure 2}

Kaplan-Meier survival curves were generated for selected immune-specific tumors (A, LUAD; B, CESC; C, SKCM; D, BRCA; E, LGG; F, KIRC). Red line represents high immune scores and blue line represents low immune scores. $P<$ 0.05 in Log-rank test. OS, overall survival in days. 

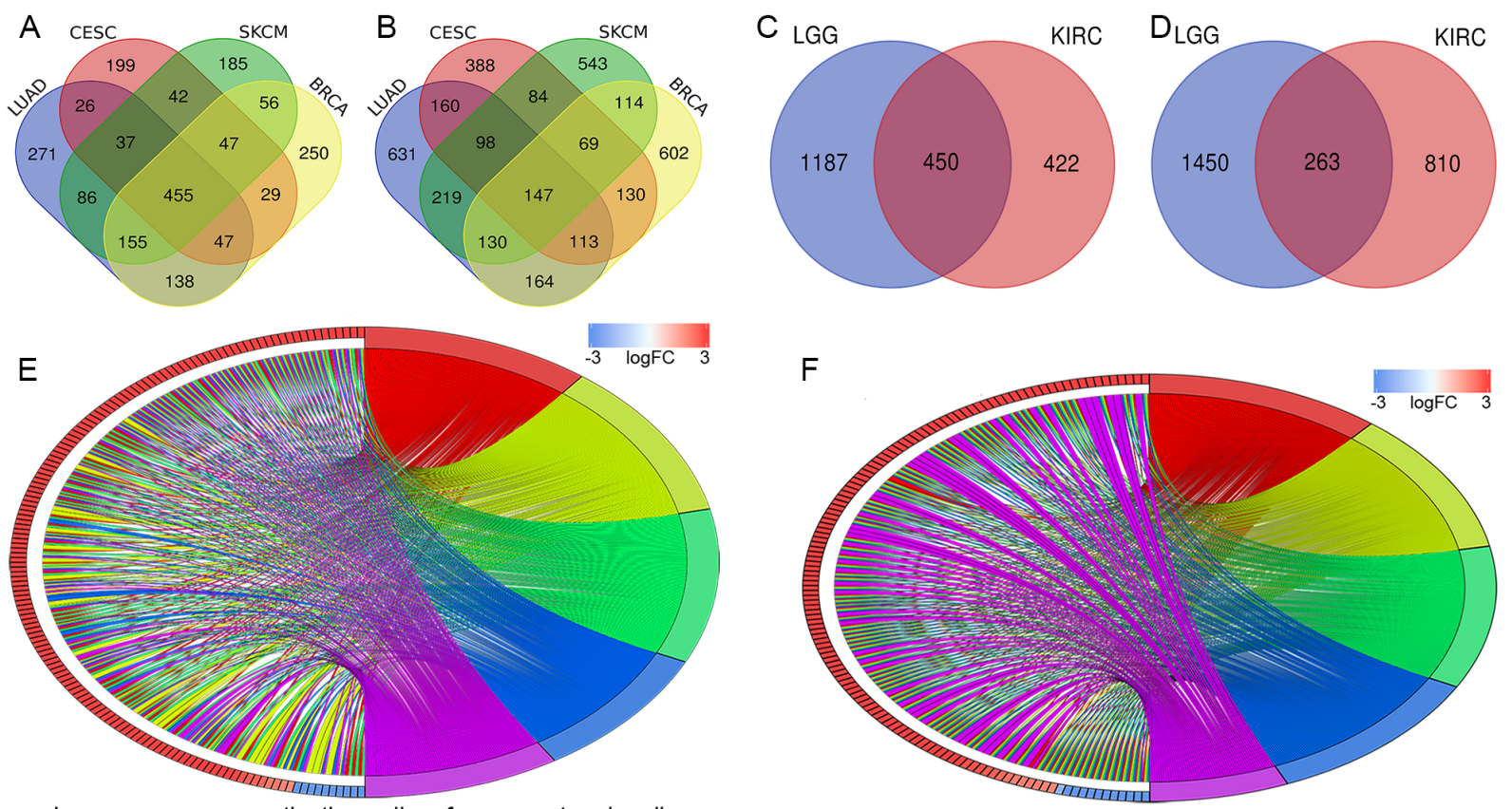

Immune response-activating cell surface receptor signaling pathway

$\square$ Regulation of immune effector process

Immune response-regulating cell surface receptor signaling

pathway

Lymphocyte mediated immunity

Adaptive immune response based on somatic recombination of immune receptors built from immunoglobulin superfamily domains

G

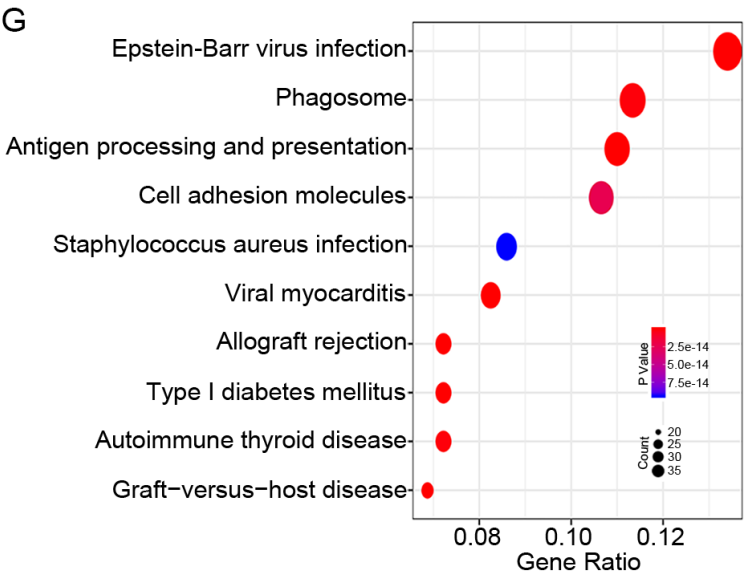

Neutrophil activation

Neutrophil degranulation

Neutrophil activation involved in immune response

Neutrophil mediated immunity

Response to interferon-gamma

$\mathrm{H}$

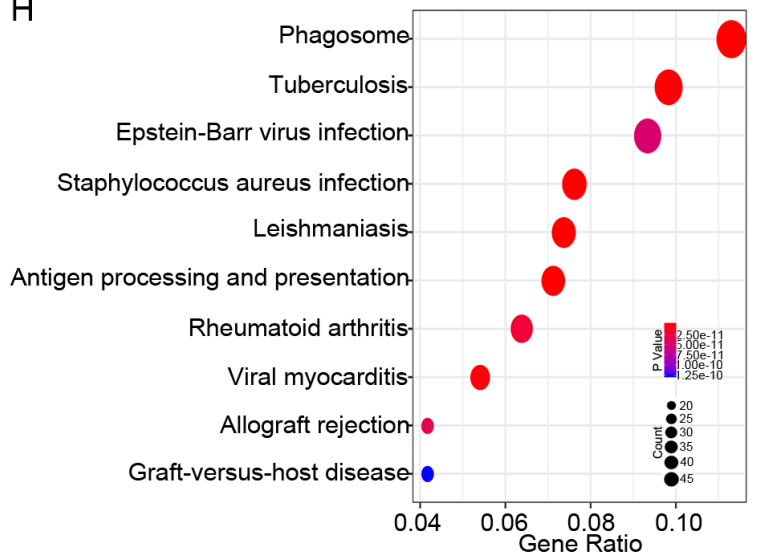

\section{Figure 3}

The functional enrichment analysis of the common DEGs in positive and negative subgroups. (A-D) Venn diagrams showing the number of common upregulated ( $A$ and $C$ ) and downregulated ( $B$ and $D$ ) DEGs in positive and negative subgroups, respectively. (E-G) Top five GO terms and top ten KEGG terms in both subgroups. 


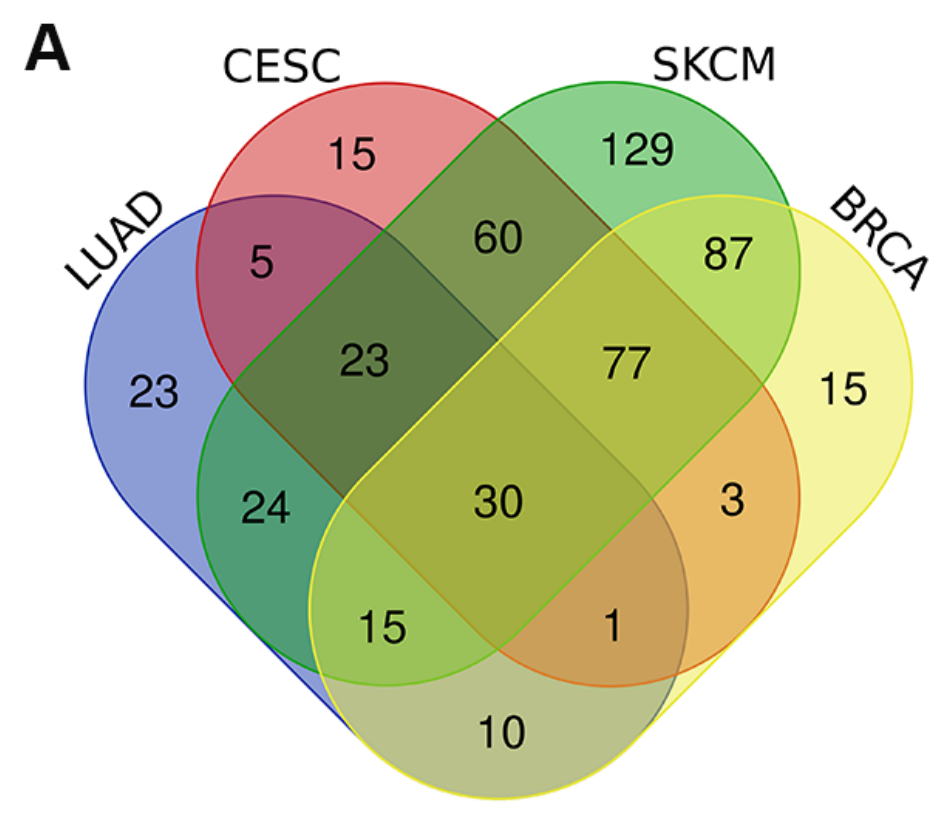

D

B

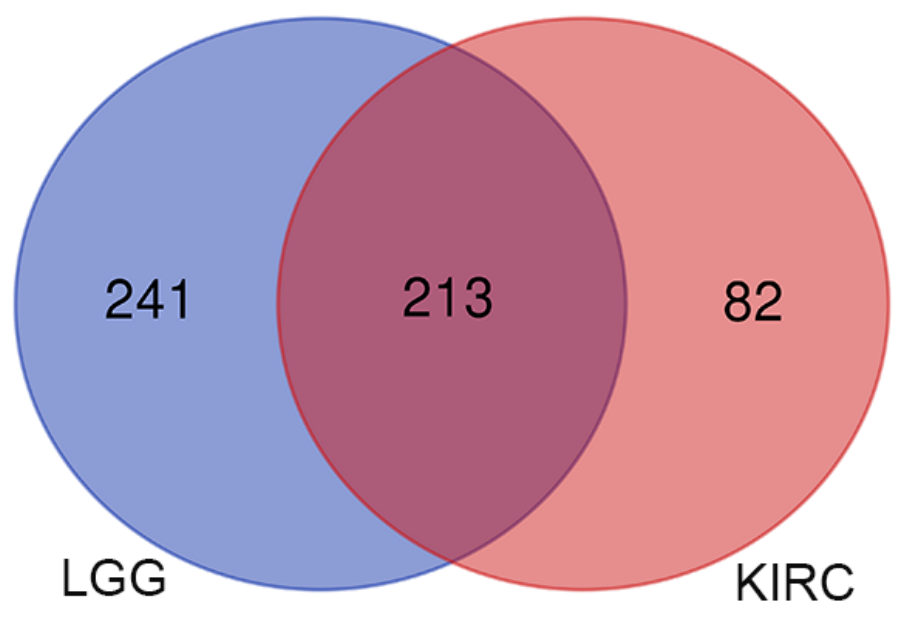

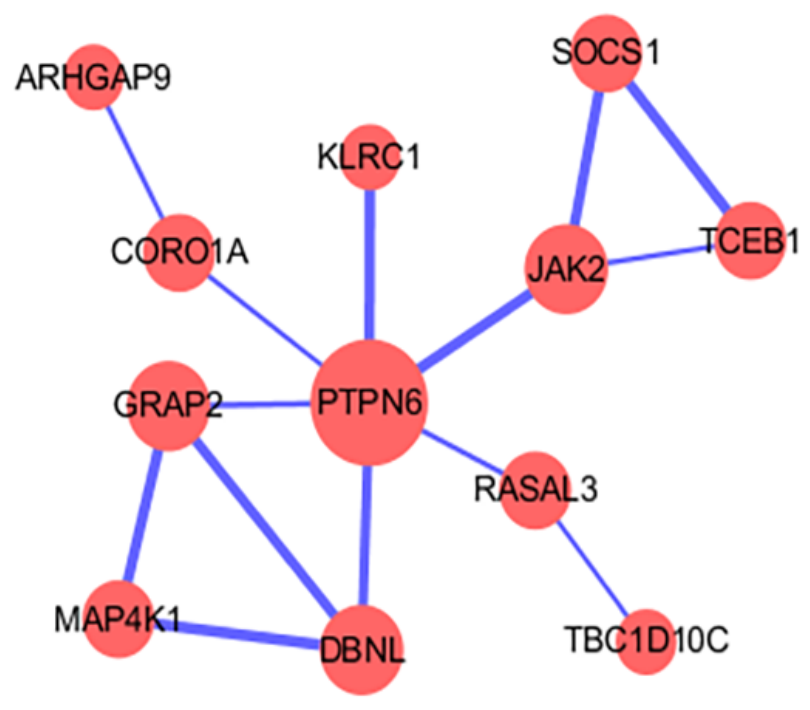

C

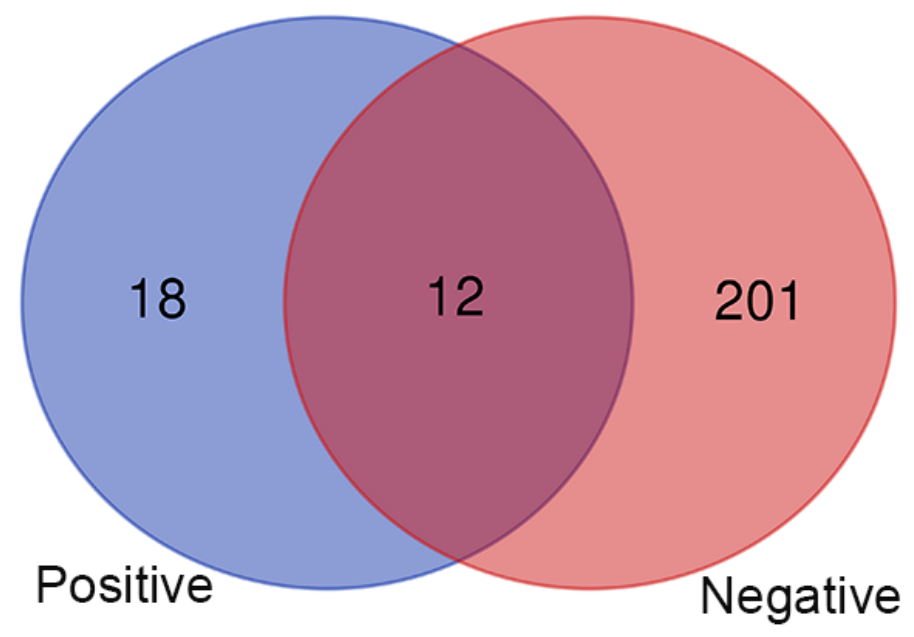

Figure 4

Identification of hub genes. (A-C) Venn diagrams showing the number of common DEGs with prognostic value in positive subgroups (A), negative subgroups (B), and both subgroups(C). (D) the twelve candidate hub genes proceed to construct the PPI network to identify the real hub genes. The size of node indicates the number of interacting proteins with the designated protein, and the thickness of edge represents the strength of interaction between proteins. 


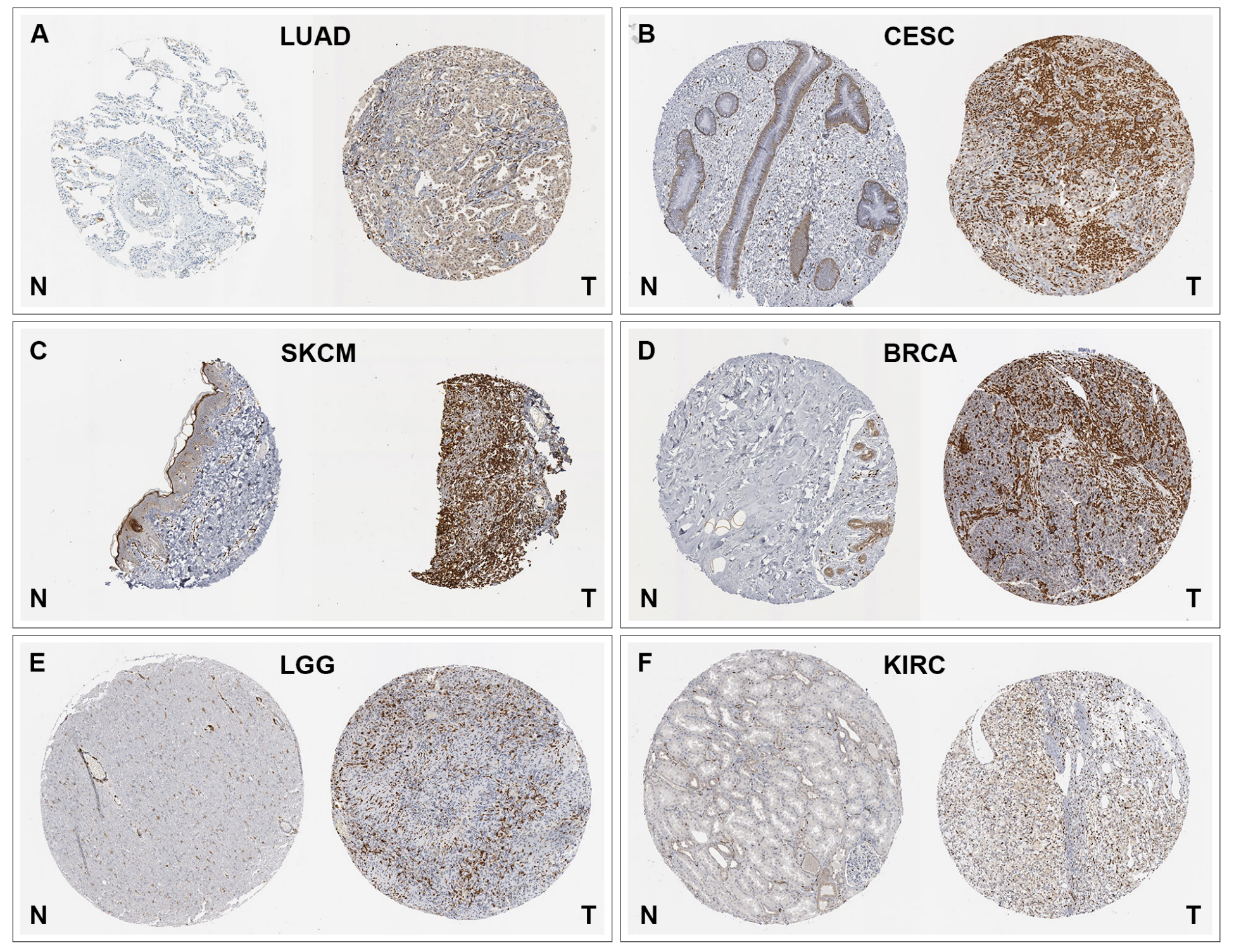

Figure 5

Validation the expression of the real hub gene on translational level by The Human Protein Atlas database. (A) LUAD. (B) CESC. (C) SKCM. (D) BRCA. (E) LGG. (F) KIRC. 
A

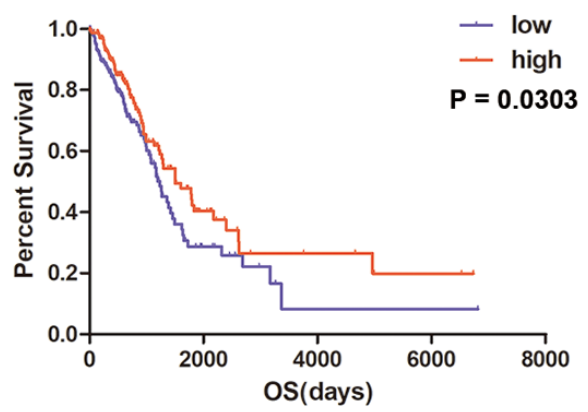

D

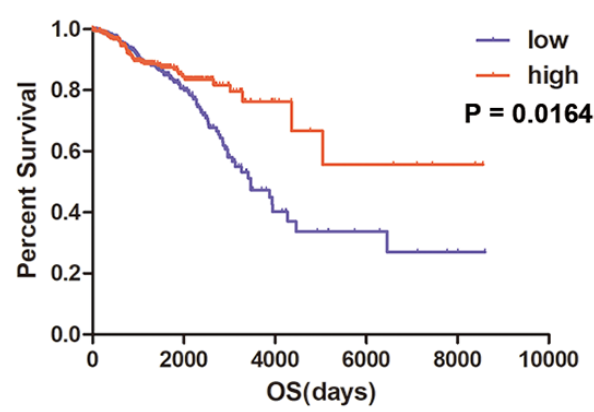

B

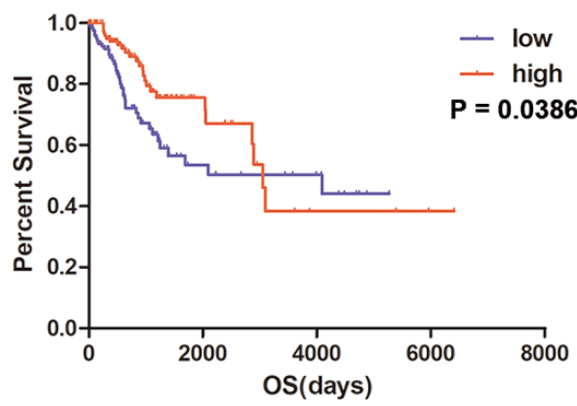

E

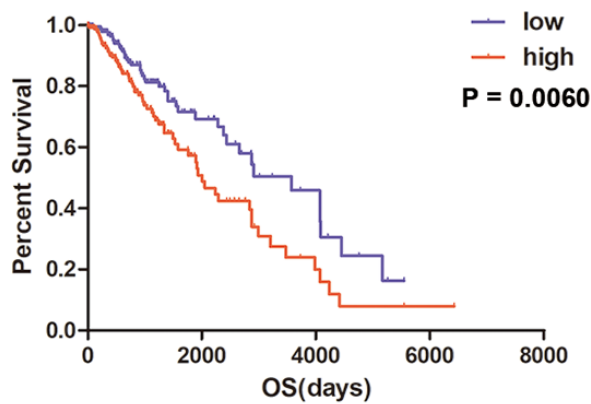

C

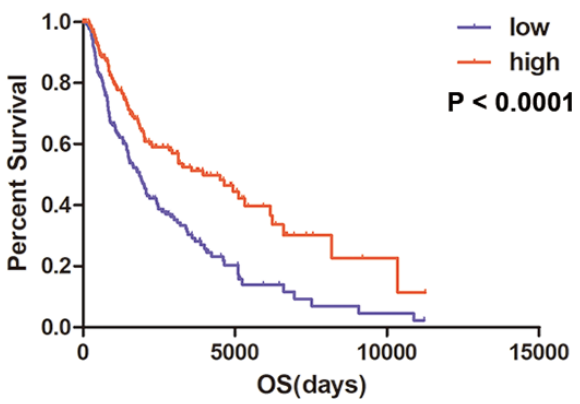

$\mathbf{F}$

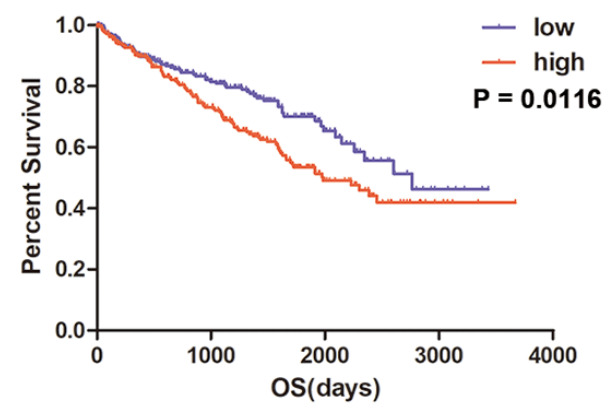

Figure 6

Validation of correlation of the real hub gene with overall survival in six cancer types. (A) LUAD. (B) CESC. (C) SKCM. (D) BRCA. (E) LGG. (F) KIRC. Red line represents high expression level of PTPN6 and blue line represents low expression. 


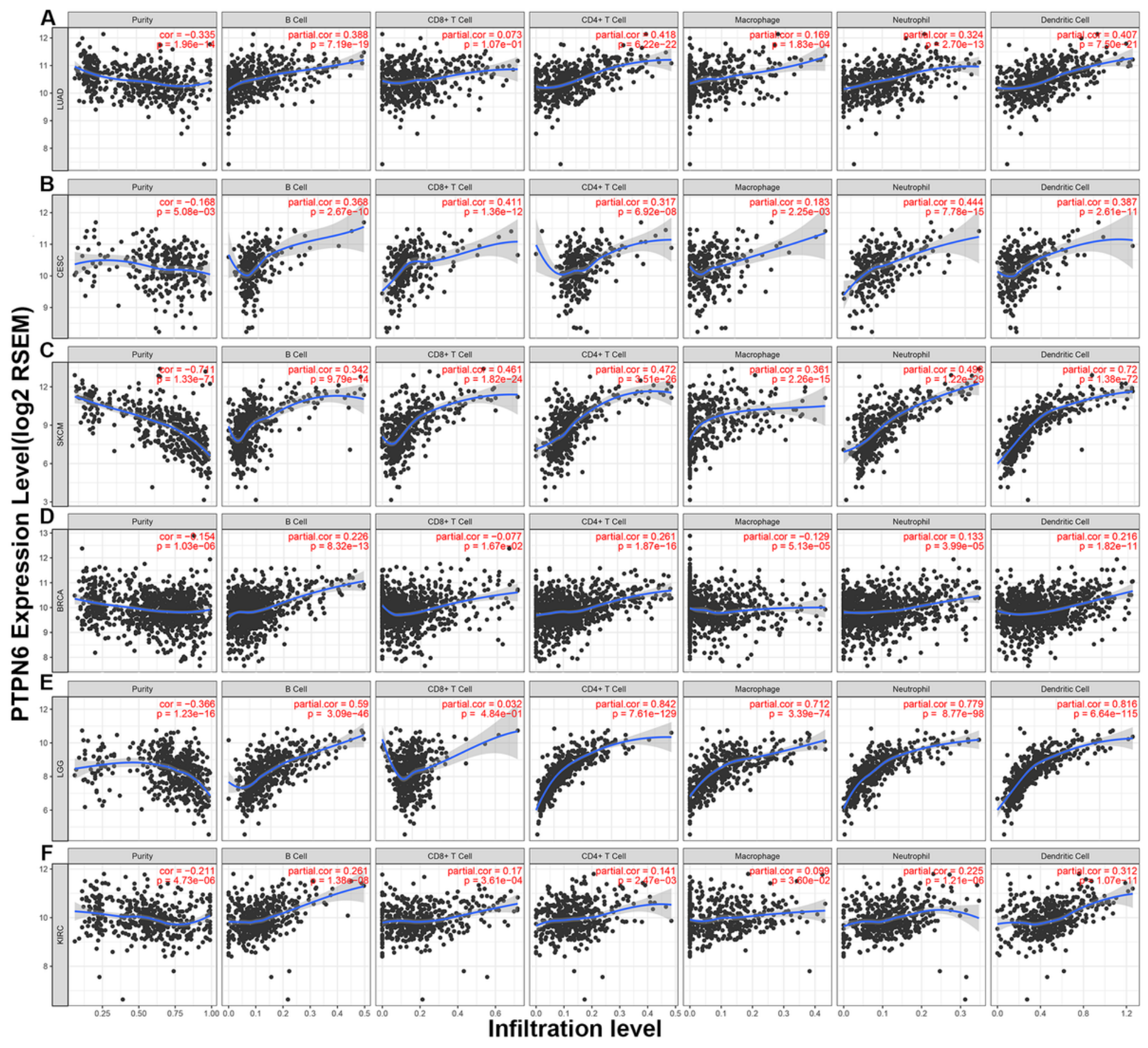

Figure 7

Correlation of PTPN6 with tumor-infiltrating immune cells in six cancer types. (A) PTPN6 expression has moderately positive correlations with infiltrating levels of B cells, CD4+ T cells, neutrophils, and dendritic cells in LUAD, but it is not associated with infiltrating levels of CD8+ T cells. (B) PTPN6 expression has moderately positive correlations with infiltrating levels of immune cells, other than macrophages in CESC. (C) A high correlation between PTPN6 and tumor-infiltrating immune cells was observed in SKCM. (D) PTPN6 expression showed a weakly positive correlation with B cells, CD4+ T cells, neutrophils, and dendritic cells, but a weakly positive correlation with CD8+ T cells and macrophages in BRCA. (E) PTPN6 expression has a significantly positive correlated with tumor-infiltrating immune cells except CD8+ T cells in LGG. (F) PTPN6 expression has a weakly positive association with infiltrating levels of B cells, CD8+ T cells, CD4+ T cells, macrophages and neutrophils in KIRC, but no significant correlation with infiltrating level of dendritic cells. 


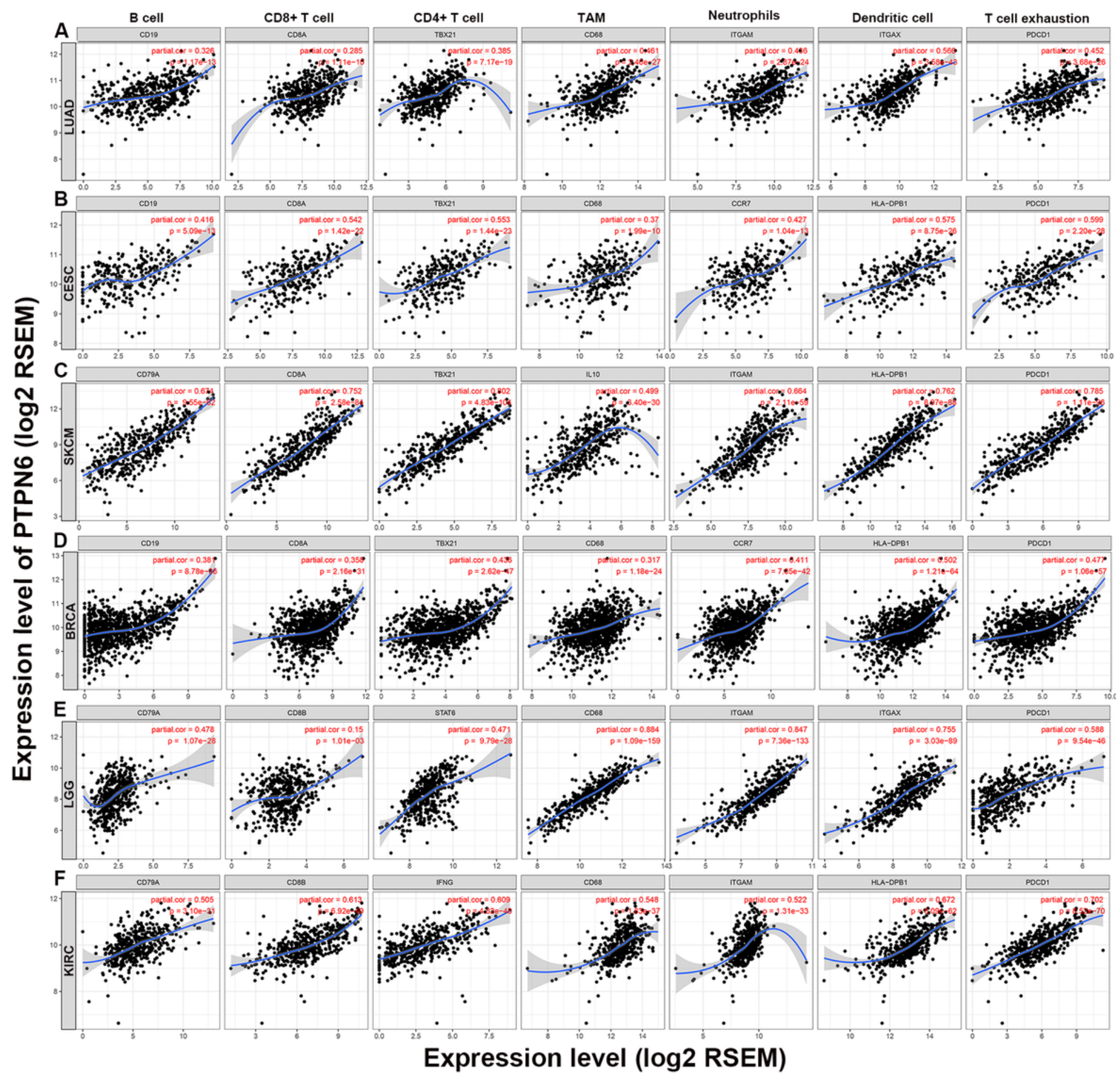

Figure 8

Correlation of PTPN6 with marker sets of diverse immune cells. (A-F) PTPN6 expression was significantly correlated with CD19 and CD79A in B cell, CD8A and CD8B in CD8+ T cell, TBX21, STAT6 and IFNG in CD4+ T cell, CD68 and IL-10 in tumor-associated macrophage, ITGAM and CCR7 in neutrophils, ITGAM and HLA-DPB1 in dendritic cell. 